\title{
Remote management of dental problems in children during and post the COVID-19 pandemic outbreak: A teledentistry approach
}

\author{
Sivakumar Nuvvula ${ }^{1, A-F}$, Sreekanth Kumar Mallineni ${ }^{1,2, A-F}$ \\ ${ }^{1}$ Department of Pediatric and Preventive Dentistry, Narayana Dental College and Hospital, Nellore, India \\ ${ }^{2}$ Department of Preventive Dental Science, College of Dentistry, Majmaah University, Al-Zulfi, Saudi Arabia \\ A - research concept and design; $\mathrm{B}$ - collection and/or assembly of data; $\mathrm{C}$ - data analysis and interpretation; \\ $D$ - writing the article; $E$ - critical revision of the article; $F$ - final approval of the article
}

Address for correspondence

Sivakumar Nuvvula

E-mail: dentist4kids@gmail.com

Funding sources

None declared

Conflict of interest

None declared

Acknowledgements

The authors would like to thank Dr. Debarchhana Jena pediatric dentist from Bengaluru, India, for sharing Fig. 1.

Received on December 13, 2020

Reviewed on February 7, 2021

Accepted on February 9, 2021

Published online on May 21, 2021

Cite as

Nuvvula S, Mallineni SK. Remote management of dental problems in children during and post the COVID-19 pandemic outbreak: A teledentistry approach. Dent Med Probl. 2021;58(2):237-241. doi:10.17219/dmp/133182

DOI

$10.17219 / \mathrm{dmp} / 133182$

Copyright

○) 2021 by Wroclaw Medical University

This is an article distributed under the terms of the

Creative Commons Attribution 3.0 Unported License (CC BY 3.0)

(https://creativecommons.org/licenses/by/3.0/)

\begin{abstract}
The recent pandemic outbreak of coronavirus disease 2019 (COVID-19) has created a helpless situation in healthcare systems worldwide. The disease can be transmitted in different ways, e.g., through contact, droplets, fomites, and aerosol-generating procedures. Subsequently, the World Health Organization (WHO) released recommendations regarding precautions to be taken by all healthcare workers, including dentists, in order to avoid the transmission of severe acute respiratory syndrome coronavirus 2 (SARS-CoV-2). COVID-19 indeed has become a pandemic, and there is a need for an innovative method to continue providing dental care to children with a minimal risk of cross-infection.

The objective of this short communication was to draw special attention to the teledentistry model in pediatric dentistry and to guide the management of children with dental problems during the COVID-19 pandemic.

Teledentistry is a feasible method and its use can overcome challenges in the present situation. The teledentistry approach enables providing guidance, treatment plans and follow-up with remote-assistance dental care through the use of information technology instead of face-to-face contact with patients.
\end{abstract}

Keywords: dentists, coronavirus, COVID-19, SARS-CoV-2, practice management 


\section{Introduction}

Teledentistry is a form of telemedicine explicitly dedicated to dentistry. Teledentistry utilizes electronic dental records, information and communication technology (ICT), digital dental photography, and the Internet for consultation, supervision as well as continuing dental education. The usage of mobile phones in teledentistry has become an attractive innovation due to an increase in availability, and improved digital photography capabilities and data processing of smartphones, which allows users to multitask, having access to affordable and secure cloud storage. The smartphone camera can be useful in dental photography, in recording the baseline preoperative oral health status, and in facilitating diagnoses and appropriate treatment plans. Since smartphones can be easily carried and used at any time, they are useful in improving patient-centered care delivery as point-of-care testing (POCT) devices. The teledentistry model can facilitate screening, data collection and primary prevention. ${ }^{1}$ With minimal training, parents, teachers or other caregivers can collect and share the required digital data from children at home or school, using user-friendly mobile technology for remote evaluation by a pediatric dentist. ${ }^{2}$

After the recent outbreak of the coronavirus disease 2019 (COVID-19) pandemic, the World Health Organization (WHO) released recommendations regarding precautions to be taken by all healthcare workers, including dentists, in order to avoid the transmission of severe acute respiratory syndrome coronavirus 2 (SARS-CoV-2). ${ }^{3}$ These refer to different transmission modes, such as contact, droplets, fomites, and aerosolgenerating procedures. ${ }^{3,4}$ As dentistry is a profession that involves face-to-face contact with patients and attendants in a closed setting, as per the current information, there are high chances of contracting this disease. ${ }^{5}$ Furthermore, almost all dental procedures involve aerosols in the dental operatory. ${ }^{4}$ The WHO also makes it clear that, based on the available data and experience, the key is to limit close contact between the infected people and others in order to break the chain of COVID-19 transmission. ${ }^{3}$ This risk is higher in pediatric dentistry, as the affected children are usually asymptomatic or have mild-to-moderate clinical symptoms of viral infection. ${ }^{4}$ Hence, community-based COVID-19 transmission may involve children from different age groups. As there are no available universal guidelines for pediatric dental procedures during the COVID-19 pandemic, routine dental procedures should be deferred, and only acute dental emergencies should be treated. In acute emergencies, pediatric dentists should be aware of the recommended protocols for managing children's dental problems. It is essential to adopt these protocols to protect themselves as well as children and their parents, at the same time preventing viral transmission. ${ }^{6}$ One of the best options to prevent viral transmission is teledentistry. It has been reported that there is high acceptance for teledentistry among patients and oral healthcare professionals. ${ }^{1,7}$ Teledentistry saves time, and shortens the distance between the dental operatory and children and their parents. Teledentistry possibly allows dental teams to examine children in their most accustomed environments, and significantly diminishes dental anxiety and fear of children and their parents or caregivers. ${ }^{7,8}$ This type of approach is very much required now to overcome the present COVID-19 pandemic crisis. Therefore, this narrative review was planned to outline the use of teledentistry in managing children's dental problems, both during and after the COVID-19 pandemic.

\section{Literature search}

An extensive search of the literature reported from December 2019 to December 2020 was conducted using the PubMed database. The keywords used in the search strategy were "COVID-19", "teledentistry", "children", "dental problems," and "management" in various combinations. In order to identify additional data published on the use of teledentistry to manage dental problems in children during COVID-19, a hand search was performed. The electronic search yielded 35 citations, and with regard to the details of the search words, the retrieved citations included: "COVID-19" AND "teledentistry" AND "children" - 1; "COVID-19" AND "teledentistry" - 30; and "teledentistry" AND "children" - 4. The hand search did not reveal any additional data. From the retrieved data, essential articles were utilized for this narrative review.

\section{Discussion}

\section{Teledentistry for pediatric dental practice}

During this COVID-19 pandemic, pediatric dentists may consider the use of teledentistry for the remote consultation, triage and delivery of dental care whenever possible and applicable. Teledentistry (virtual dental visits or dental care at a distance) enables pediatric dentists to meet various dental care requirements while avoiding close contact with their pediatric patients. However, teledentistry can only be practiced according to the respective national or state authorities'/associations' guidelines. ${ }^{8}$ Teledentistry encompasses various components, such as consultation, diagnosis, triage, and monitoring. Teleconsultation is the most common form of teledentistry through which parents, caregivers or school teachers can seek advice for children who require a dental appointment. It may also help with the continuation of the treatment plan and follow-up advice during the quarantine or lockdown period. Teletriage consists in 
the safe, correct and timely evaluation of the child's symptoms through remote consultation, using the pediatric dentist's smartphone or laptop computer. Similarly, parents can also use their smartphones to take pictures of their children's teeth and to provide information to the pediatric dentist. Triage involves distinguishing between emergency and non-emergency dental needs, and between those that require prioritization or deferment. In telediagnosis, the smartphone can be utilized to exchange information and photographs taken as directed by the pediatric dentist, thereby facilitating the diagnosis of emergencies related to early childhood caries (ECC), dental pain, traumatic dental injuries (TDI), and facial swelling. Telemonitoring (virtual visits) can substitute regular physical visits in routine monitoring of disease progression or treatment outcomes. ${ }^{9}$

\section{Benefits and barriers related to pediatric teledentistry}

The benefits include the ability to provide primary and specialized dental care, improved communication between the dental team and children/parents, and the effective triage of patients. ${ }^{2}$ Thus, teledentistry reduces inappropriate referrals and waiting time, aids in timely diagnoses, treatment provision and follow-up, and lessens the costs related to travel and accommodation. Teledentistry might improve clinical outcomes and contribute to a reduction in pain and other comorbidities associated with delayed diagnosis and treatment. It supports monitoring a child's oral health and implementing preventive measures in such pandemic situations. ${ }^{2}$ The possible barriers for teledentistry include the level of acceptance of its use by children, parents and pediatric dentists. The pediatric dentist may be concerned about making an inappropriate diagnosis and incurring additional expenses related to infrastructure, equipment and high-speed Internet. The issues related to poor Internet access, the lack of technical training and the lack of expertise can be causes for concern for the dental team and parents/caregivers. As teledentistry is new to the healthcare system, the concerns related to insufficient financial reimbursement, scanty guidelines, and the coordination between children, accompanying personnel and the dental team are other challenges. ${ }^{10}$ The persons involved might have to depend on other people or government aid to utilize teledentistry in dental practice in communities without smartphones and Internet facilities. ${ }^{2,9}$

Teledentistry could aid the dental community by covering a broader geographical area, enabling more efficient functioning and helping many people in need while maintaining social distancing. The most recent systematic review confirms that teledentistry is a viable option for remote consultation, screening, diagnosis, treatment planning, and dentistry mentoring. ${ }^{1}$ It also states that the rapidly developing ICT has greatly improved the cost-efficiency, precision and effectiveness of remote assistance for pediatric dentists. ${ }^{1}$ There is high acceptance for teledentistry among clinicians and patients alike. ${ }^{11,12}$ Parents have to be trained in video calling, taking the needed intraoral pictures and transferring them to the pediatric dentist by means of smartphones. Pediatric dentists have several possible approaches available for telecommunication with children and their parents, guardians and caregivers (Fig. 1). Through pediatric teledentistry, consultation with children and parents may be possible in 2 ways: real-time consultation (synchronous) or consultation through the stored records (asynchronous). Teleconsultation and teledentistry will impact the way the insurance company and the patient are billed. One may also want to consider accessing patient management software to review the patient's chart and radiographs. Such a review can be done via the virtual remote login applications using the software, private telenetworks or social media, including Skype, WhatsApp, Instagram, and Messenger.

A systematic review by a Brazilian research group suggested that teledentistry could improve the quality of care related to the diagnosis and management of oral lesions, and shorten distances for patients who need specialized diagnoses and specialists providing care. ${ }^{13}$ Another systematic review from the USA concluded that teledentistry could be comparable to one-to-one dentistry for oral screening in remote areas, areas with limited access to care, long-term care facilities, and as part of school-based programs. ${ }^{14}$ The authors also stated that teleconsultation was possible and valid in detecting oral health issues. ${ }^{14}$ Consequently, Daniel et al. performed a systematic qualitative review and reported that the rapidly emerging communication technologies and information systems progressively revealed improvement in the cost-effeciency, precision and effectiveness of remote assistance for oral healthcare professionals. ${ }^{15}$

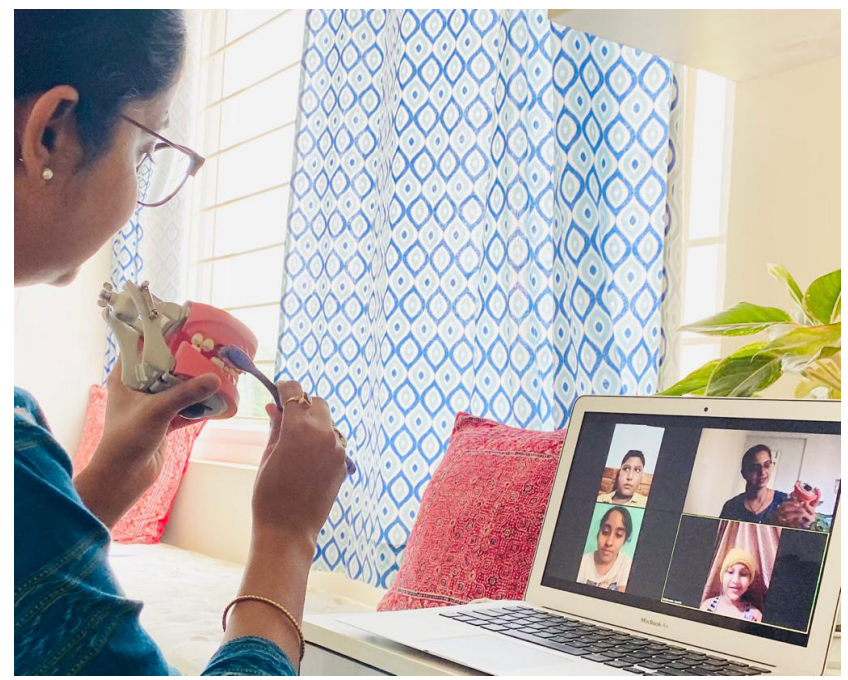

Fig. 1. Pediatric dentist providing remote oral hygiene instructions to children through teledentistry 
Alabdullah et al. found that the exposure of pediatric dental students to the teledentistry model increased. ${ }^{16} \mathrm{Re}$ garding the records used in teledentistry, an Australian group reviewed photographic examinations and found that the image analysis provided accuracy comparable to visual inspection in diagnosing dental problems. ${ }^{17}$ The authors concluded that the results were least comparable between photographic and visual inspection techniques in the case of enamel defects. ${ }^{17}$ In the present COVID-19 pandemic situation, it is challenging to provide regular dental treatment to children. A Saudi Arabian crosssectional survey was performed on undergraduate students and it was found that the study subjects knew very little about teledentistry. ${ }^{18}$ The author suggested the inclusion of teledentistry as a continuing dental education topic. ${ }^{18}$ Pereira et al. reported that teledentistry was an emerging tool to help maintain contact with the patient without any risk of the transmission of infections such as COVID-19. ${ }^{19}$ A British study opined that healthcare professionals should consider adapting patient pathways and using telehealth as a consultation method to recover services and reduce the spread of COVID-19. ${ }^{20}$ A cross-sectional survey by Surdu and Langelier found an increase in the utilization of oral health services for children, especially in rural areas. ${ }^{21}$

Furthermore, various authors worldwide preferred utilizing teledentistry in this pandemic period. ${ }^{22-27}$ There is a shortage of specialists and a lack of holistic oral healthcare for children due to the closure of academic institutes and private practices. Hence, through the pediatric teledentistry approach, the number of dental consultations for children could be increased by enhancing specialists' accessibility to remote communities and otherwise inaccessible areas. The flowchart presented in Fig. 2 depicts a simple approach to teledentistry for children, including the use of various social media and communicators, decision-making for emergency vs nonemergency dental needs, triage, decisions on home care instructions, and mobile or portable dentistry at home vs treatment at the dental operatory/dental clinic based on the needs of the individual child.

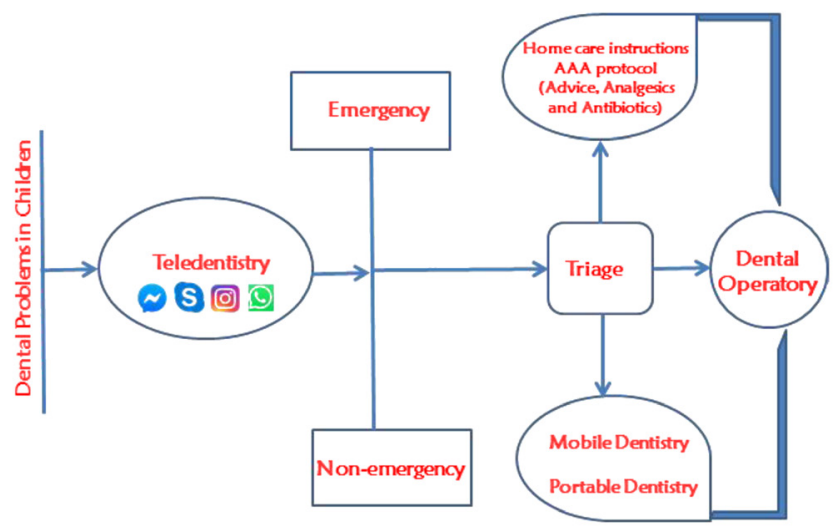

Fig. 2. Proposed teledentistry model to deal with dental problems in children during and post COVID-19 pandemic

\section{Conclusions}

Teledentistry for children could be considered as pediatric teledentistry. Pediatric dentists should train themselves and their teams also with regard to technical issues. Communication and the provision of treatment through teledentistry requires additional time and care to ensure proper advice to children and parents. Pediatric teledentistry could be a viable option for managing dental problems in children during pandemics.

\section{ORCID iDs}

Sivakumar Nuvvula (1) https://orcid.org/0000-0002-1204-5551 Sreekanth Kumar Mallineni (i) https://orcid.org/0000-0002-9432-2590

\section{References}

1. Irving $M$, Stewart $R$, Spallek $H$, Blinkhorn A. Using teledentistry in clinical practice as an enabler to improve access to clinical care: A qualitative systematic review. J Telemed Telecare. 2018;24(3):129-146. doi:10.1177/1357633X16686776

2. Estai M, Kruger E, Tennant M, Bunt S, Kanagasingam Y. Challenges in the uptake of telemedicine in dentistry. Rural Remote Health. 2016;16(4):3915.

3. Peng $X, X u X, L i$, Cheng $L$, Zhou X, Ren B. Transmission routes of 2019-nCoV and controls in dental practice. Int J Oral Sci. 2020;12(1):9. doi:10.1038/s41368-020-0075-9

4. Mallineni SK, Innes NP, Raggio DP, Araujo MP, Robertson MD, Jayaraman J. Coronavirus disease (COVID-19): Characteristics in children and considerations for dentists providing their care. Int J Paediatr Dent. 2020;30:245-250. doi:10.1111/ ipd.12653

5. Gambarini G, Galli M, Gambarini E, et al. Fine aerosols and perceived risk of COVID-19 among Italian dental practitioners: An experimental survey. J Contemp Dent Pract. 2020;21(6):599-603.

6. Estai $M$, Kanagasingam $Y$, Mehdizadeh $M$, et al. Teledentistry as a novel pathway to improve dental health in school children: A research protocol for a randomised controlled trial. BMC Oral Health. 2020;20:11. doi:10.1186/s12903-019-0992-1

7. Mariño R, Ghanim A. Teledentistry: A systematic review of the literature. J Telemed Telecare. 2013;19(4):179-183. doi:10.1177/1357633×13479704

8. Ferrazzano GF, Ingenito A, Cantile T. COVID-19 disease in children: What dentists should know and do to prevent viral spread. The Italian point of view. Int J Environ Res Public Health. 2020;17(10):3642. doi:10.3390/ijerph17103642

9. Mallineni SK, Bhumireddy JC, Nuvvula S. Dentistry for children during and post COVID-19 pandemic outbreak. Child Youth Serv Rev. 2021;120:105734. doi:10.1016/j.childyouth.2020.105734

10. Giudice A, Barone S, Muraca D, et al. Can teledentistry improve the monitoring of patients during the Covid-19 dissemination? A descriptive pilot study. Int J Environ Res Public Health. 2020;17(10):3399. doi:10.3390/ijerph17103399

11. Bhumireddy J, Mallineni SK, Nuvvula S. Challenges and possible solutions in dental practice during and post COVID-19. Environ Sci Pollut Res Int. 2021;28(2):1275-1277. doi:10.1007/s11356-020-10983-x

12. Royal College of Dental Surgeons of Ontario. 2020. COVID-19: Guidance for the use of teledentistry. https://www.rcdso.org/ en-ca/rcdso-members/2019-novel-coronavirus/covid-19---emergency-screening-of-dental-patients-using-teledentistry. Accessed December 13, 2020.

13. da Costa Flores AP, Lazaro SA, Molina-Bastos CG, et al. Teledentistry in the diagnosis of oral lesions: A systematic review of the literature. J Am Med Inform Assoc. 2020;27(7):1166-1172. doi:10.1093/ jamia/ocaa069

14. Alabdullah JH, Daniel SJ. A systematic review on the validity of teledentistry. Telemed J E Health. 2018;24(8):639-648. doi:10.1089/ tmj.2017.0132

15. Daniel SJ, Wu L, Kumar S. Teledentistry: A systematic review of clinical outcomes, utilization and costs. J Dent Hyg. 2013;87(6):345-352. 
16. Alabdullah JH, Van Lunen BL, Claiborne DM, Daniel SJ, Yen CJ, Gustin TS. Application of the unified theory of acceptance and use of technology model to predict dental students' behavioral intention to use teledentistry. J Dent Educ. 2020;84(11):1262-1269. doi:10.1002/jdd.12304

17. Meurer MI, Caffery LJ, Bradford NK, Smith AC. Accuracy of dental images for the diagnosis of dental caries and enamel defects in children and adolescents: A systematic review. J Telemed Telecare. 2015;21(8):449-458. doi:10.1177/1357633X15605225

18. Aboalshamat KT. Awareness of, beliefs about, practices of, and barriers to teledentistry among dental students and the implications for Saudi Arabia vision 2030 and coronavirus pandemic. J Int Soc Prev Community Dent. 2020;10(4):431-437. doi:10.4103/jispcd. JISPCD_183_20

19. Pereira LJ, Pereira CV, Murata RM, Pardi V, Pereira-Dourado SM. Biological and social aspects of Coronavirus Disease 2019 (COVID-19) related to oral health. Braz Oral Res. 2020;34:e041. doi:10.1590/1807-3107bor-2020.vol34.0041

20. Rahman N, Nathwani S, Kandiah T. Teledentistry from a patient perspective during the coronavirus pandemic. Br Dent J. 2020:1-4. doi:10.1038/s41415-020-1919-6

21. Surdu S, Langelier M. Teledentistry: Increasing utilisation of oralhealth services for children in rural areas. J Telemed Telecare. 2020:1357633X20965425. doi:10.1177/1357633X20965425

22. Martins MD, Carrard VC, Dos Santos CM, Hugo FN. COVID-19 - Are telehealth and tele-education the answers to keep the ball rolling in dentistry? Oral Dis. 2020. doi:10.1111/odi.13527

23. Maspero C, Abate A, Cavagnetto D, El Morsi M, Fama A, Farronato M. Available technologies, applications and benefits of teleorthodontics. A literature review and possible applications during the COVID-19 pandemic. J Clin Med. 2020;9(6):1891. doi:10.3390/jcm9061891

24. Sa Y, Lin WS, Morton D, Huang C. Coronavirus disease 2019 (COVID-19): Experiences and protocols from the Department of Prosthodontics at the Wuhan University. J Prosthet Dent. 2020;S0022-3913(20)30374-7. doi:10.1016/j.prosdent.2020.06.004

25. Talla PK, Levin L, Glogauer M, Cable C, Allison PJ. Delivering dental care as we emerge from the initial phase of the COVID-19 pandemic: Teledentistry and face-to-face consultations in a new clinical world. Quintessence Int. 2020;51(8):672-677. doi:10.3290/j.qi.a44920

26. Yadav V, Kumar V, Sharma S, Chawla A, Logani A. Palliative dental care: Ignored dimension of dentistry amidst COVID-19 pandemic. Spec Care Dentist. 2020;40(6):613-615. doi:10.1111/scd.12517

27. Sycinska-Dziarnowska M, Paradowska-Stankiewicz I. Dental challenges and the needs of the population during the Covid-19 pandemic period. Real-time surveillance using Google trends. Int J Environ Res Public Health. 2020;17(23):8999. doi:10.3390/ijerph17238999 